\title{
Overview About Lipid Structure
}

\author{
Rodrigo Valenzuela B. and Alfonso Valenzuela B.
}

Additional information is available at the end of the chapter

http://dx.doi.org/10.5772/52306

\section{Introduction}

The term lipid is used to classify a large number of substances having very different physical - chemical characteristics, being its solubility in organic non-polar solvents the common property for their classification. Lipids are composed of carbon, hydrogen and oxygen atoms, and in some cases contain phosphorus, nitrogen, sulfur and other elements. In this context, fats and oils are the main exponents of lipids present in foods and in nutritional processes [1,2], being diverse fatty acids and cholesterol the most representative molecules due their important metabolic and nutritional functions [3,4]. The structural, metabolic and nutritional importance of lipids in the body is supported by numerous investigations in different biological models (cellular, animals and humans). Lipids have been instrumental in the evolution of species, having important role in the growth, development and maintenance of tissues [5,6]. A clear example of this importance is the elevated fatty acid concentration present in nerve tissue, especially very long-chain polyunsaturated fatty acids $[7,8]$.

Fatty acids are, among lipids, of crucial relevance in the structure and physiology of the body because: i) forms an integral part of phospholipids in cell membranes; ii) are the primary source of energy ( $9 \mathrm{kcal} / \mathrm{g}$ or $37.62 \mathrm{kjoules} / \mathrm{g}$ ); iii) in infants, provide more than $50 \%$ of the daily energy requirements; iv) some fatty acids are of essential character and are required for the synthesis of eicosanoids and docosanoids (of 20 and 22 carbon atoms, respectively), such as leukotrienes, prostaglandins, thromboxanes, prostacyclins, protectins and resolvins), and; v) some of them may act as second messengers and regulators of gene expression $[9,10]$. Besides fatty acids, cholesterol is another lipid that has important functions in the body, among which are: i) together with phospholipids is important in the formation of cell membranes; ii) constitutes the skeleton for the synthesis of steroid hormones (androgens and estrogens); iii) from its structure is derived the structure of vitamin $\mathrm{D}$, and; iv) participates in the synthesis of the bile salts and the composition of bile secretion [11]. 
Lipids play a key role in the growth and development of the organism, where the requirements of these molecules (mainly fatty acids) will change depending on the age and physiological state of individuals [12]. Furthermore, lipids have crucial participation both, in the prevention and/or in the development of many diseases, especially chronic noncommunicable diseases [13], affecting the lipid requirements in humans [14]. As food components, lipids are also important because: i) are significant in providing organoleptic characteristics (palatability, flavor, aroma and texture); ii) are vehicle for fat soluble vitamins, pigments or dyes and antioxidants, and; iii) may act as emulsifying agents and/or promote the stability of suspensions and emulsions [15].

Fats and oils, the most common lipids in food, are triacylglyceride mixtures, i.e. structures formed by the linking of three different or similar fatty acids to the tri-alcohol glycerol [16]. A fat is defined as a mixture of triacylglycerides which is solid or pasty at room temperature (usually $20^{\circ} \mathrm{C}$ ). Conversely, the term oil corresponds to a mixture of triglycerides which is liquid at room temperature. In addition to triacylglycerides, which are the main components of fats and oils (over 90\%), these substances frequently contain, to a lesser extent, diacylglycerides, monoacylglycerides, phospholipids, sterols, terpenes, fatty alcohols, carotenoids, fat soluble vitamins, and many other minor chemical structures $[17,18]$. This chapter deals with the general aspects of lipids, especially those related to the chemical structure and function of these molecules.

\section{Fatty acids}

Fatty acids are hydrocarbon structures (containing carbon and hydrogen atoms) formed by four or more carbons attached to an acidic functional group called carboxyl group. The chemical and physical properties of the different fatty acids, such as their solubility in nonpolar solvents and the melting point, will depend on the number of carbon atoms of the molecule. [19]. The higher the number of carbon atoms of the chain the higher will be melting point of the fatty acid. According to the chain length fatty acids are referred as short-chain fatty acids, those having four (C4) to ten (C10) carbons; as medium-chain fatty acids those having twelve (C12) to fourteen (C14) carbons; long-chain fatty acids to those of sixteen (C16) to eighteen carbons (C18); and very long-chain fatty acids those having twenty (C20) or more carbon atoms. Molecules having less than four carbon atoms (C2; acetic acid and C3; propionic acid) are not considered fatty acids due their high water solubility. On the other side, fatty acids of high number of carbon atoms are not frequent, however are present in significant amount in the brain of vertebrates, including mammals and human. In the human brain have been identified fatty acids as long as 36 carbon atoms [20].

The link between carbons in fatty acids, correspond to a covalent bond which may be single (saturated bond) or double (unsaturated bond). The number of unsaturated bonds in the same molecules can range from one to six double bonds. Thus, the more simple classification of the fatty acids, divided them in those that have not double bonds, named saturated fatty acids (SAFA), and fatty acids that have one or more double bonds, collectively named unsaturated fatty acids. In turn, when the molecule has one unsaturation 
it is classified as monounsaturated fatty acid (MUFA) and when has two to six unsaturations are classified as polyunsaturated fatty acids (PUFAs) [21]. The presence of unsaturation or double bonds in fatty acids is represented by denoting the number of carbons of the molecule followed by an indication of the number of double bonds, thus: C18:1 corresponds to a fatty acid of 18 carbons and one unsaturation, it will be a MUFA. C20: 4 correspond to a molecule having 20 carbons and four double bonds, being a PUFA. Now, it is necessary to identify the location of the unsaturations in the hydrocarbon chain both in MUFAs and PUFAs [22].

\section{Nomenclature of fatty acids}

According to the official chemical nomenclature established by IUPAC (International Union of Practical and Applied Chemistry) carbons of fatty acids should be numbered sequentially from the carboxylic carbon (C1) to the most extreme methylene carbon (Cn), and the position of a double bond should be indicated by the symbol delta $(\Delta)$, together to the number of the carbon where double bonds begins. According to this nomenclature: C18: 1, $\Delta 9$, indicates that the double bond is between carbon 9 and 10 [23]. However, in the cell the metabolic utilization of fatty acids occurs by the successive scission of two carbon atoms from the $\mathrm{C} 1$ to the $\mathrm{Cn}$ (mitochondrial or peroxisomal beta oxidation). This means that as the fatty acid is being metabolized (oxidized in beta position), the number of each carbon atom will change, creating a problem for the identification of the metabolic products formed as the oxidation progress. For this reason R. Holman, in 1958, proposed a new type of notation that is now widely used for the biochemical and nutritional identification of fatty acids [24]. This nomenclature lists the carbon enumeration from the other extreme of the fatty acid molecule. According to this notation, the $\mathrm{C} 1$ is the carbon farthest from the carboxyl group (called as terminal or end methylene carbon) which is designed as "n", " $\omega$ " or "omega". The latter notation is the most often used in nutrition and refers to the last letter of the Greek alphabet [25]. Thus, C18: $1 \Delta 9$ coincidentally is C18: $1 \omega$ 9 in the " $\omega$ " notation, but C18: $2 \Delta 9, \Delta 12$, according to this nomenclature $\omega$ would be C18: 2 $\omega-6$. What happens with fatty acids having more than a double bond? Double bonds are not randomly arranged in the fatty acid structure. Nature has been "ordained" as largely incorporate them in well-defined positions. Most frequently double bonds in PUFAs are separated by a methyl group (or most correctly methylene group) forming a $-\mathrm{C}=\mathrm{C}-\mathrm{C}-\mathrm{C}=\mathrm{C}-$ structure which is known as "unconjugated structure", which is the layout of double bonds in most naturally occurring PUFAs [26]. However, although much less frequently, there are also present "conjugated structures" where double bonds are not separated by a methylene group, forming a $-\mathbf{C}=\mathbf{C}-\mathbf{C}=\mathbf{C}$ - structure. This particular structural disposition of double bonds, i.e conjugated structures, is now gaining much interest because some fatty acids having these structures show special nutritional properties, they are called "conjugated fatty acids". Most of them are derived from the unconjugated structure of linoleic acid $(\mathrm{C} 18: 2 \omega-6)[27,28]$.

For the application of the " $\omega$ " nomenclature and considering the "order" of double bonds in unsaturated fatty acids having unconjugated stucture, it can be observed that by 
pointing the location of the first double bond, it will automatically determined the location of the subsequent double bonds [29]. Thus, C18: $1 \omega-9$, which has a single double bond at $\mathrm{C} 9$ counted from the methyl end, correspond to oleic acid (OA), which is the main exponent of the $\omega-9$ family. Oleic acid is highly abundant both in vegetable and animal tissues. C18: $2 \omega-6$ corresponds to a fatty acid having double bonds at the C6 and C9 (for unconjugated fatty acids it is not necessary to indicate the position of the second or successive double bonds). This is linoleic acid (LA), the main exponent of the $\omega-6$ family and which is very abundant in vegetable oils and to a lesser extent in animal fats [30]. C18: 3, $\omega-3$ corresponds to a fatty acid having double bonds at C3, C6 and C9. It is alpha-linolenic acid (ALA), the leading exponent of the $\omega-3$ family. ALA is a less abundant fatty acid, almost exclusively present in the vegetable kingdom and specifically in land-based plants [31]. Within (LCPUFAs), C20: 4, $\omega-6$ or arachidonic acid (AA); C20: 5, $\omega-3$ or eicosapentaenoic acid (EPA) and; C22 : 6, $\omega-3$ or docosahexaenoic acid (DHA), are of great nutritional importance and are only found in ground animal tissues (AA) and in aquatic animal tissues (AA, EPA and DHA) and in plants of marine origin (EPA and DHA) [32].

The increase of double bonds in fatty acids significantly reduces its melting point. Thus, for a structure of the same number of carbon atoms, if it is saturated may give rise to a solid or semisolid product at room temperature, but if the same structure is unsaturated, may originate a liquid or less solid product at room temperature. Figure 1 shows the classification of fatty acids according to their degree of saturation and unsaturation and considering the notation " $\omega$ ", and table 1 shows different fatty acids, showing the C nomenclature, their systematic name, their common name and the respective melting point.

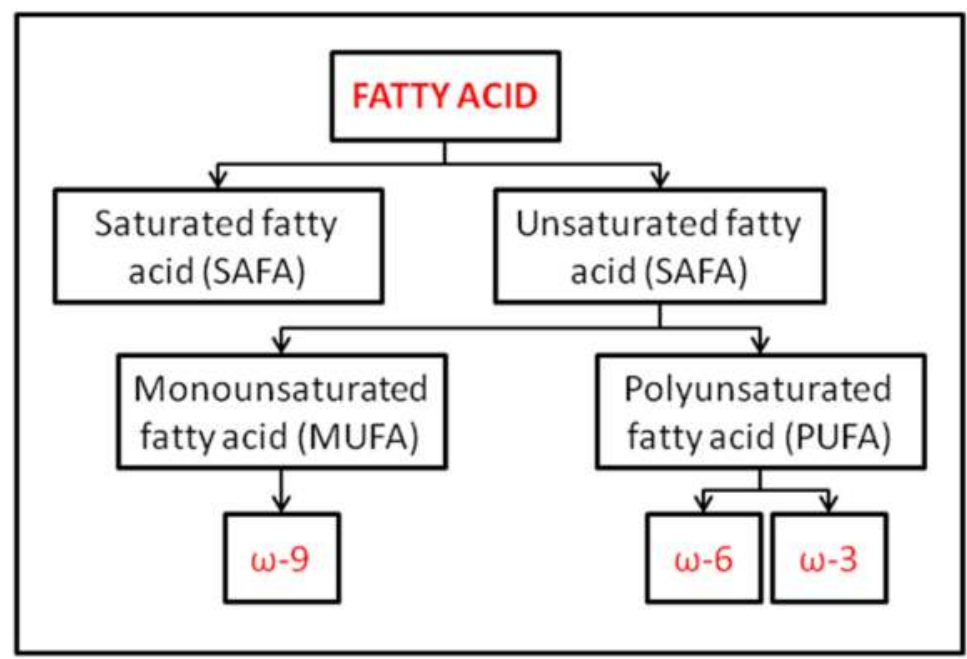

Figure 1. Classification of fatty acids according to their degree of saturation and unsaturation and considering the notation " $\omega "$. 


\begin{tabular}{|c|c|c|c|}
\hline Nomenclature & Systematic name & Common name & Melting point ${ }^{\circ} \mathrm{C}$ \\
\hline \multicolumn{4}{|c|}{ Saturated Fatty Acid } \\
\hline C 4:0 & Butanoic & Butiric & -5.3 \\
\hline C 6:0 & Hexaenoic & Caproic & -3.2 \\
\hline C 8:0 & Octanoic & Caprilic & 16.5 \\
\hline C10:0 & Decanoic & Capric & 31.6 \\
\hline C12:0 & Dodecanoic & Lauric & 44.8 \\
\hline C14:0 & Tetradecanoic & Miristic & 54.4 \\
\hline $\mathrm{C} 16: 0$ & Hexadecanoic & Palmitic & 62.9 \\
\hline C18:0 & Octadecanoic & Estearic & 70.1 \\
\hline C20:0 & Eicosanoic & Arachidic & 76.1 \\
\hline $\mathrm{C} 22: 0$ & Docosanoic & Behenic & 80.0 \\
\hline C24:0 & Tetracosanoic & Lignocenic & 84.2 \\
\hline \multicolumn{4}{|c|}{ Unsaturated Fatty Acid } \\
\hline C16:1 & 9-Hexadececoic & Palmitoleic & 0.0 \\
\hline C18:1 & 9-Octadecenoic & Oleic & 16.3 \\
\hline C18:1 & 11-Octodecenoic & Vaccenic & 39.5 \\
\hline C18:2 & 9,12-Octadecadienoic & Linoleic & -5.0 \\
\hline C18:3 & 9,12,15-Octadecatrienoic & Linolenic & -1.0 \\
\hline C20:4 & 5,8,11,14-Eicosatetraenoic & Arachidonic & 49.5 \\
\hline
\end{tabular}

Table 1. Different fatty acids, showing the $\mathrm{C}$ nomenclature, their systematic name, their common name and the respective melting point.

\section{Mono-, di- and triacylglycerides}

The structural organization of fatty acids in food and in the body is mainly determined by the binding to glycerol by ester linkages. The reaction of a hydroxyl group of glycerol, at any of its three groups, with a fatty acid gives rise to a monoacylglyceride. The linking of a second fatty acid, which may be similar or different from the existing fatty acid, gives rise to a diacylglyceride. If all three hydroxyl groups of glycerol are linked by fatty acids, then this will be a triacylglyceride [33]. Monoacylglycerides, by having free hydroxyl groups (two) are relatively polar and therefore partially soluble in water. Different monoacylglycerides linked to fatty acids of different lengths are used as emulsifiers in the food and pharmaceutical industry [34]. The less polar diacylglycerides which have only one free hydroxyl group are less polar than monoacylglycerides and less soluble in water. Finally, triacylglycerides, which lack of free hydroxyl groups are completely non-polar, but highly soluble in non-polar solvents, which are frequently used for their extraction from vegetable or animal tissues, because constitutes the energy reserve in these tissues [35]. Diacylglycerides and monoacylglycerides are important intermediates in the digestive and absorption process of fats and oils in animals. In turn, some of these molecules also perform other metabolic functions, such as diacylglycerides which may act as "second messengers" at the intracellular level and are also part of the composition of a new generation of oils nutritionally designed as "low calorie oils" [36]. When glycerol forms mono-, di-, or 
triacylglycerides, its carbon atoms are not chemically and structurally equivalent. Thus, carbon 1 of the glycerol is referred as carbon $(\alpha)$, or sn-1 (from "stereochemical number"); carbon 2 is referred as carbon $(\beta)$, or sn-2, and carbon 3 as $(\gamma)$, or sn-3. It is important to note

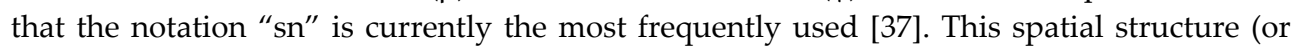
conformation) of mono-, di- and triacyglycerides is relevant in the digestive process of fats and oils (ref). Figure 2 shows the structure of a monoacylglceride, a diacylglyceride and a triacylglyceride, specifying the "sn-" notation.

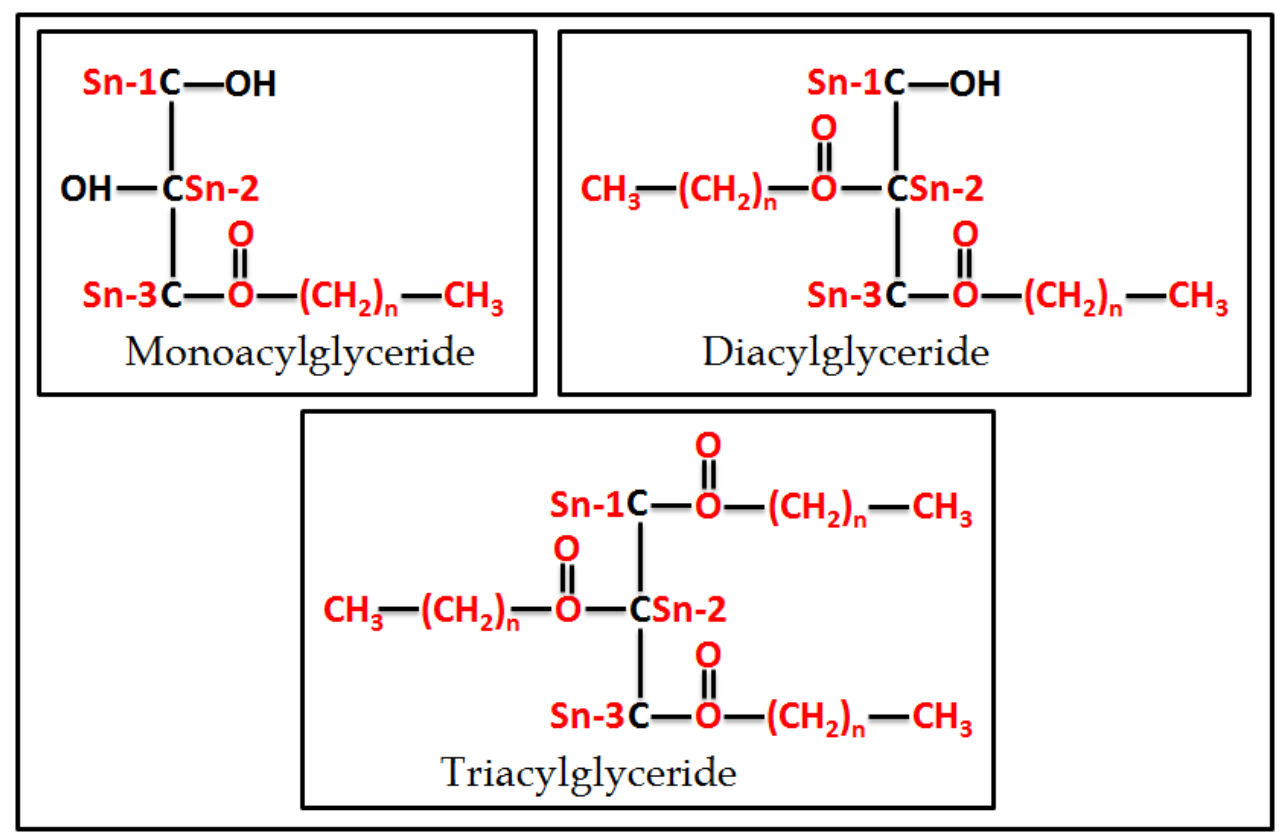

Figure 2. Structure of a monoacylglyceride, a diacylglyceride and a triacylglyceride, specifying the "sn-" notation

\section{Essential fatty acids}

The capability of an organism to metabolically introduce double bonds in certain positions of a fatty acid or the inability to do this, determines the existence of the so-called nonessential or essential fatty acids (EFAs). According to this capability, mammals, including primates and humans, can introduce a double bond only at the $\mathrm{C} 9$ position of a saturated fatty acid (according to " $\omega$ " nomenclature) and to other carbons nearest to the carboxyl group, but not at carbons nearest the $\mathrm{C} 1$ position [38]. This is the reason why OA is not an EFA. In contrast, mammals can not introduce double bonds at $\mathrm{C} 6$ and $\mathrm{C} 3$ positions, being the reason why AL and ALA are EFAs. By derivation, the AA is formed by the elongation and desaturation of LA, and EPA and DHA, which are formed by elongation and desaturation of ALA, become also essential for mammals when their respective precursors (LA and ALA, respectively) are nutritionally deficient [39]. Figure 3 shows the chemical 
structure of a SAFA, such as the stearic acid (C18:0), AO, LA and ALA, exemplifying the " $\omega "$ notation of each and indicating the essential condition in relation to the position of their unsaturated bonds.

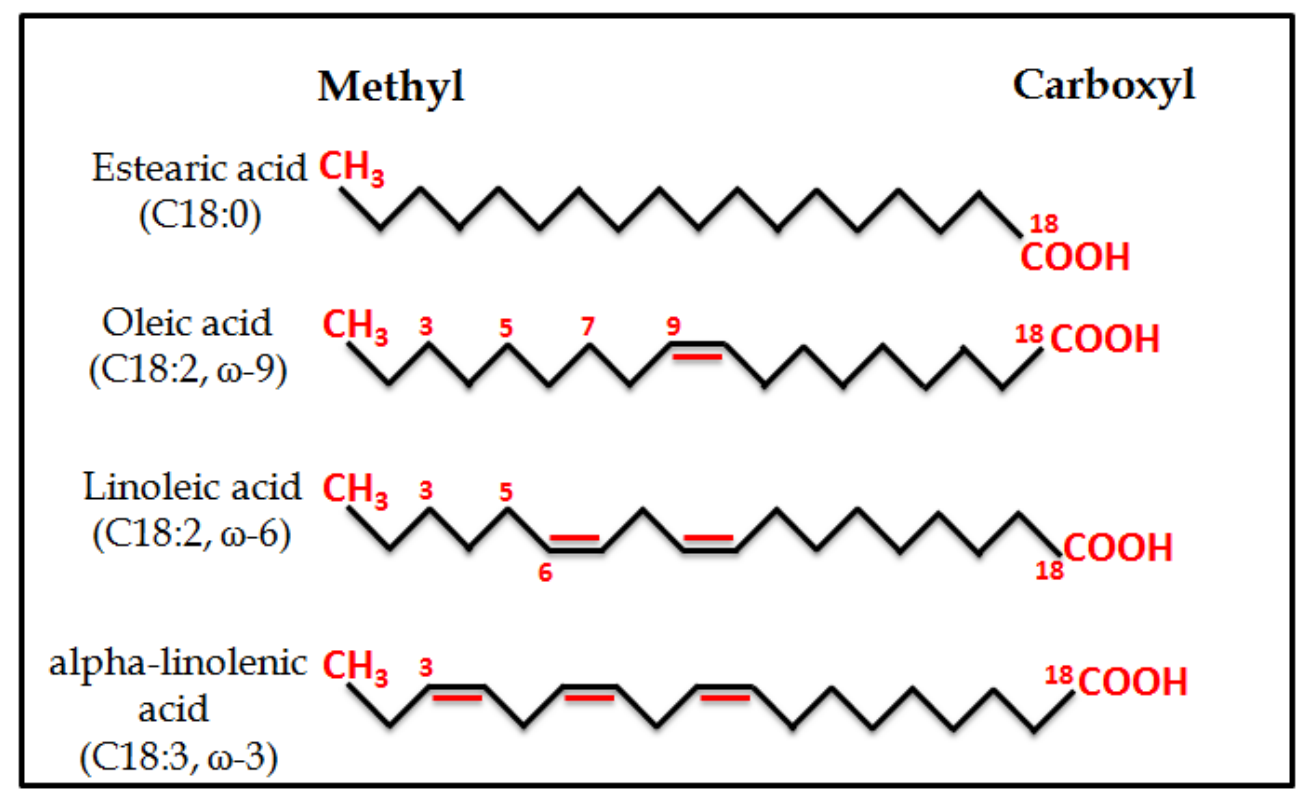

Figure 3. The chemical structure of a SAFA, such as the stearic acid (C18:0), AO, LA and ALA, exemplifying the " $\omega$ " notation of each and indicating the essential condition in relation to the position of their unsaturated bonds

\section{Isomerism of fatty acids}

According to the distribution of double bonds in a fatty acid and to its spatial structure, unsaturated fatty acids may have two types of isomerism: geometrical isomerism and positional isomerism. By isomerism it is referred to the existence two or more molecules having the same structural elements (atoms), the same chemical formula and combined in equal proportions, but having a different position or spatial distribution of some atoms in the molecule [40].

\subsection{Geometrical isomers of fatty acids}

Carbon atoms forming the structure of the fatty acids possess a three-dimensional spatial structure which forms a perfect tetrahedron. However, when two carbons having tetrahedral structure are joined together through a double bond, the spatial conformation of the double bond is modified adopting a flat or plane structure [41]. Rotation around single bonds $(C-C)$ is entirely free, but when they are forming a double bond $(C=C)$, this rotation is impeded and the hydrogen atoms that are linked to each carbon involved in the bond may 
be at the same side or opposed in the plane forming the double bond. If hydrogen atoms remain at the same side, the structure formed is referred as cis isomer (denoted as " $c$ "). When hydrogen atoms remain at opposite sides the structure formed is referred as trans isomer (denoted as " $t$ ", trans: means crossed) [42]. Figure 4 shows the cis - trans geometric isomerism of fatty acids. The cis or trans isomerism of fatty acids confers them very different physical properties, being the melting point one of the most relevant [43]. Table 2 shows the melting point of various cis - trans geometric isomers of different fatty acids. It can be observed substantial differences in the melting point of cis- or trans isomers for the same fatty acid. Melting point differences bring to the geometrical isomers of a fatty acid very different biochemical and nutritional behavior. Fatty acids having trans isomerism, especially those of technological origin (such as generated during the partial hydrogenation of oils), have adverse effect on humans, particularly referred to the risk of cardiovascular diseases [44]. It is noteworthy that the majority of naturally occurring fatty acids have cis isomerism, although thermodynamically is more stable the trans than the cis isomerism, whereby under certain technological manipulations, such as the application of high temperature (frying process) or during the hydrogenation process applied for the manufacture of shortenings, cis isomers are easily transformed into trans isomers [45].

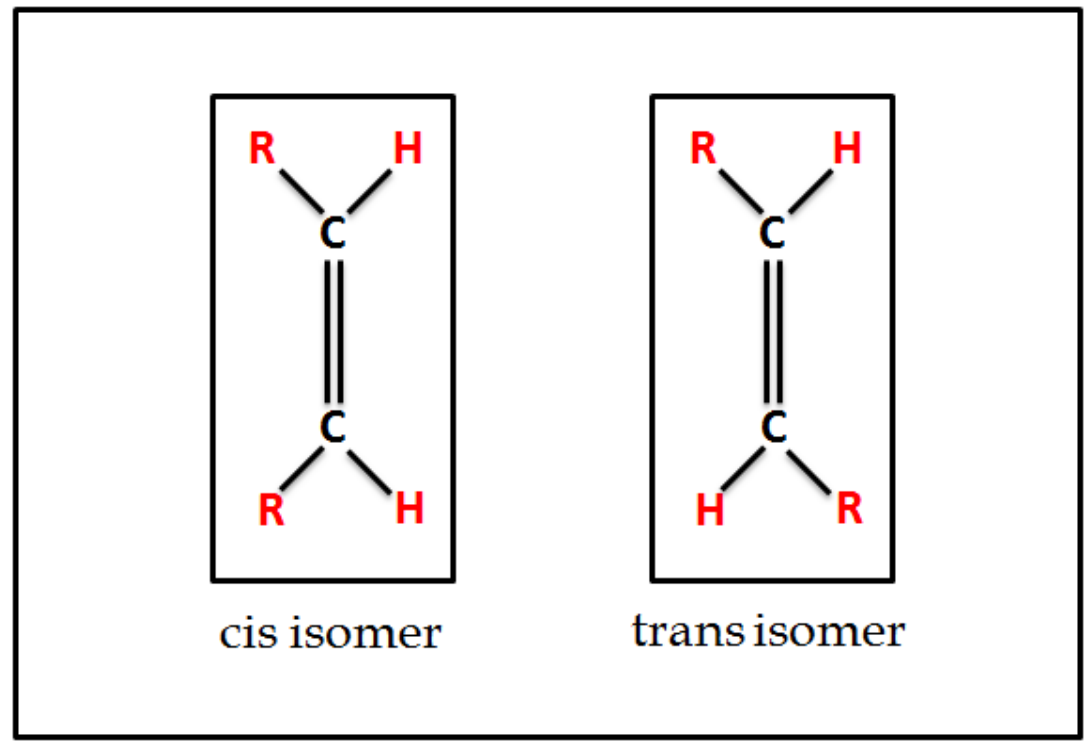

Figure 4. Geometric isomerism of fatty acids

\subsection{Positional isomers of fatty acids}

Positional isomerism refers to the different positions that can occupy one or more double bonds in the structure of a fatty acid. For example, oleic acid (C18:1 $\Delta 9 \mathrm{c})$, is a common fatty acid in vegetable oils, particularly in olive oil, but vaccenic acid (C18:1 $\Delta 11 \mathrm{t}$ ) is more common in animal fats. This is a double example, since both fatty acids are geometric 
isomers (oleic acid cis and vaccenic acid trans) and at the same time positional isomers, since oleic acid has a double bond at the $\Delta 9$ position and vaccenic acid at the $\Delta 11$ position [46].

\begin{tabular}{|ccc|}
\hline Fatty acid & Isomerism & Melting point $\left({ }^{\circ} \mathrm{C}\right)$ \\
\hline C12:0 & ----- & 44.2 \\
C16:0 & ----- & 62.7 \\
C18:0 & ----- & 69.6 \\
C18:1 & Cis & 13.2 \\
C18:1 & Trans & 44.0 \\
C18:2 & cis, cis & -5.0 \\
C18:2 & trans, trans & 18.5 \\
C18:3 & cis, cis, cis & 11.0 \\
C20:3 & trans, trans, trans & 29.5 \\
\hline
\end{tabular}

Table 2. Changes in the melting point of various cis - trans geometrical isomers of different fatty acids

In general, all fatty acids naturally present positional isomerism of their more frequent molecular structure. However, these isomers occur in very low concentrations. Unlike the known biochemical and nutritional effects of trans geometric isomers, there is little information about the biological effects of positional isomers and for the majority of them these effects are considered as not relevant, except for some conjugated structures, such as conjugated linoleic acid (C18:2, $\Delta 9, \Delta 11, \mathrm{CLA})$, a geometric and positional isomer of the most common linoleic acid, for which it has been attributed various health properties, especially those related to anti-inflammatory and lipolytic actions, but up to date the scientific evidence for these properties are considered insufficient [47]. Such as geometrical isomerism, the technological manipulation of fatty acids (i.e. temperature and/or hydrogenation) increases the number and complexity of the positional isomers [48]. Figure 5 summarizes the positional and geometric isomers of unsaturated fatty acids.

\section{Phospholipids}

Phospholipids are minor components in our diet because less than $4-5 \%$ of our fat intake corresponds to phospholipids. However, this does not detract nutritionally important to these lipids, since they are important constituents of the cellular structure having also relevant metabolic functions [49]. Life, in its origin, would not have been possible without the appearance of phospholipids, as these structures are the fundamental components of all cellular membranes. Phospholipids have structural and functional properties that distinguish them from their counterparts, triacylglycerides. In phospholipids positions sn-1 and sn-2 of the glycerol moiety are occupied by fatty acids, more frequently polyunsaturated fatty acids, linked to glycerol by ester bonds. The sn-3 position of glycerol is linked to orthophosphoric acid [50]. The structure which is formed, independent of the type of fatty acid that binds at sn-1 and sn-2, is called phosphatidic acid. The presence of phosphate substituent at the sn-3 position of the glycerol gives a great polarity to this part of the molecule, being non-polar the rest of the structure, such as in triacylglycerides. This 
double feature, a polar extreme and a non-polar domain due the presence of the two fatty acids characterizes phospholipids as amphipathic molecules (amphi: both; pathos: sensation) [51].

\section{Geometric}
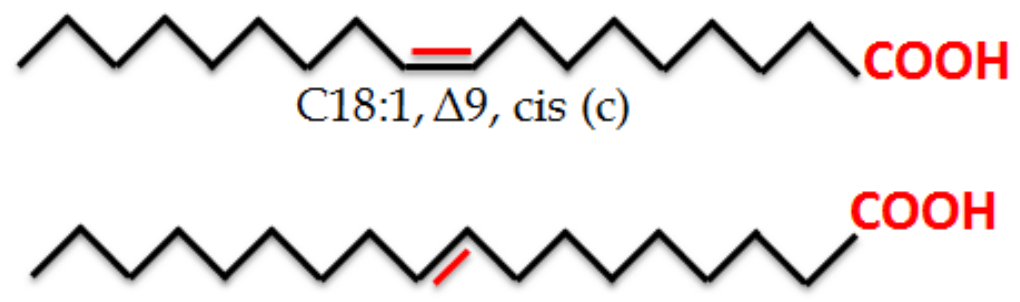

\section{$\mathrm{C} 18: 1, \Delta 9$ trans $(\mathrm{t})$}

\section{Positional}
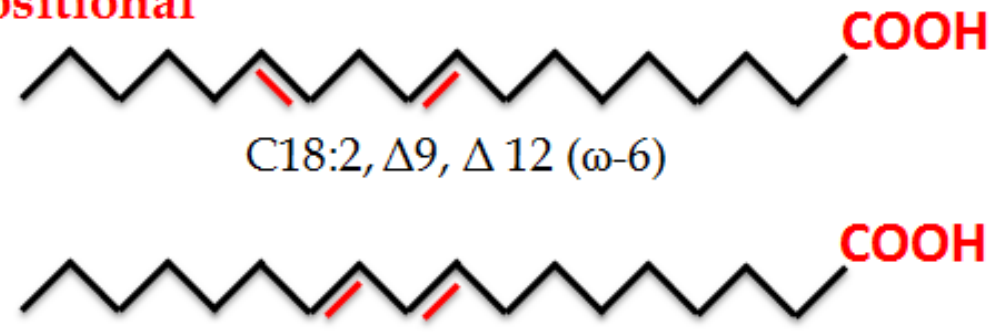

C18:2, $\Delta 9, \Delta 11$ (conjugated structure)

Figure 5. Positional and geometric isomers of unsaturated fatty acids

The structure of phospholipids is usually simplified representing the polar end as a sphere and the fatty acids as two parallel rods. Figure 6 shows the chemical structure of phosphatidic acid in its simplified representation. The amphipathic character of phosphatidic acid can be increased by joining to the phosphate different basic and polar molecules that increases the polarity to the extreme of the sn-3 position. When the substituent of the phosphate group is the aminoacid serine it is formed phosphatidylserine; when it is etanolamine it is formed phosphatidylethanolamine (frequently known as cephalin); when choline is the substituent it is formed phosphatidylcholine (well known as lecithin); and when the substituent is the polyalcohol inositol it is formed phosphatidylinositol, a very important molecule involved in cell signaling. [52]. 


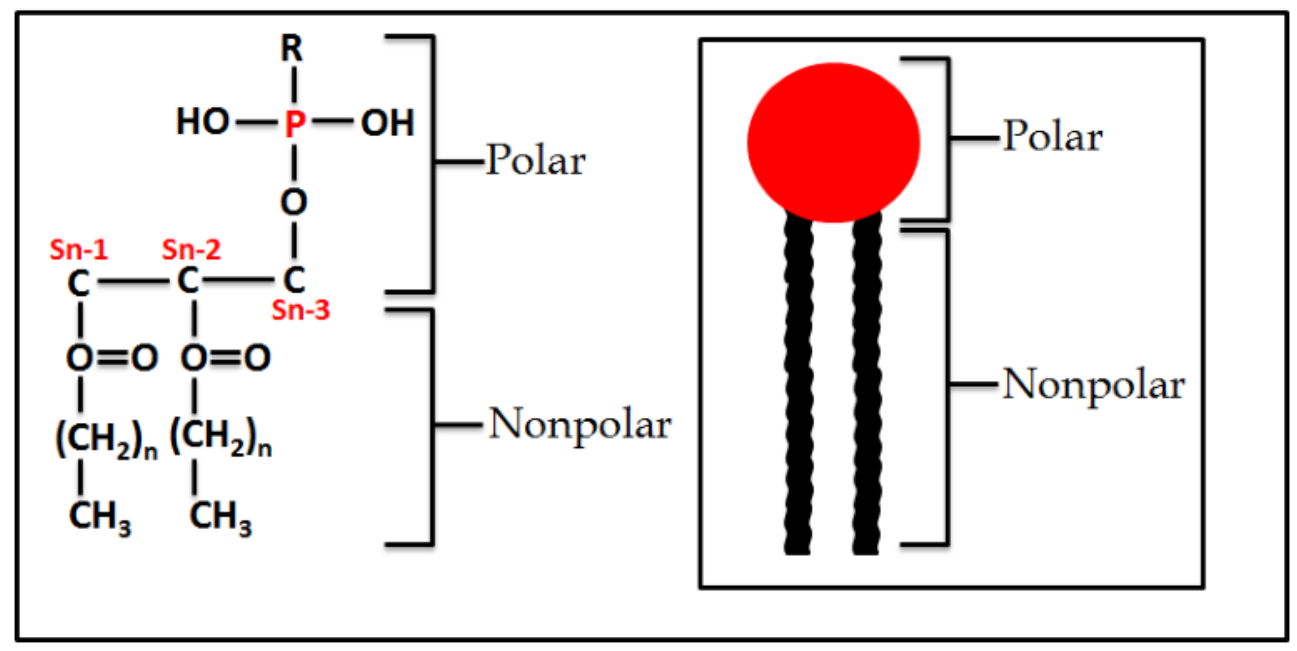

Figure 6. Chemical structure of phosphatidic acid and its simplified representation

These more complex phospholipids are much more common than phosphatidic acid, since this is only the structural precursor of the above molecules. Figure 7 shows the structure of various phospholipids. A number of other molecules are also classified as phospholipids, but are structurally different. Cardiolipin is a "double" phospholipid in which two phosphatidic acid molecules are attached through their phosphates by a molecule of glycerol. Cardiolipin is a very important in the structure of the inner membrane of mitochondria and due their molecular volume it is the only immunogenic phospholipid (which stimulates the formation of antibodies) [53]. Plasmalogens are other lipid molecules related to phospholipids. In these molecules the substituent at sn-1 position of the glycerol is not a fatty acid, but a fatty alcohol which is linked to glycerol by an ether linkage. Phosphatidalethanolamine (different than phosphatidylethanolamine) is an abundant plasmalogen in the nervous tissue [54]. Phosphatidalcholine, the plasmalogen related to phosphatidylcholine, is abundant in the heart muscle. Another structures related to phospholipids are sphingolipids. In these structures glycerol is replaced by the amino alcohol; sphingosine. When the hydroxyl group (alcoholic group) of sphingosine is substituted by phosphocholine, it is formed sphingomyelin, which is the only sphingolipid that is present in significant amount in human tissues as a constituent of myelin that forms nerve fibers [ref]. Platelet activating factor (PAF) is an unusual glycerophospholipid structure. In this molecule position sn-1 of glycerol is linked to a saturated alcohol through an ether bond (such as in plasmalogens) and at the sn- 2 binds an acetyl group instead of a fatty acid. PAF is released by a variety of cells and by binding to membrane receptors produces aggregation and degranulation of platelets, has potent thrombotic and inflammatory effects, and is a mediator of anaphylactic reactions [55]. 


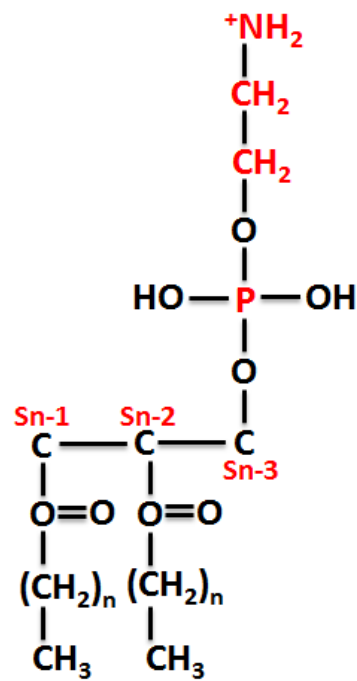

Phosphatidylethanolamine<smiles>CCOC(=O)[C@H](O[PH](O)(O)OCC(N)C(=O)O)[C@@H]([SeH])C(=O)OCC</smiles>

Phosphatidylserine<smiles>CCOC(=O)[C@H]([SeH])[C@@H](OCC)O[PH](O)(O)OCC[N+](C)(C)C</smiles>

Phosphatidalcholine

Figure 7. Structure of various phospholipids

A fundamental aspect of phospholipids is their participation in the structure of biological membranes, and the structural characteristics of the fatty acids are relevant to determine the behavior and the biological properties of the membrane. As an example, a diet rich in saturated fatty acids result in an increase in the levels of these fatty acids into cell membrane phospholipids, causing a significant decrease in both, membrane fluidity and in the ability of these structure to incorporate ion channels, receptors, enzymes, structural proteins, etc., effect which is associated to an increased cardiovascular risk [56]. By contrast, a diet rich in monounsaturated and/or polyunsaturated fatty acids produce an inverse effect. At the nutritional and metabolic level this effect is highly relevant because as the fatty acid composition of the diet is directly reflected into the fatty acid composition of phospholipids, changes in the composition of the diet, i.e. increasing the content of polyunsaturated fatty acids, will prevent the development of several diseases [57]. Figure 8 shows a simulation how the structural differences of the fatty acids which comprise phospholipids may affect the physical and chemical behavior of a membrane.

\section{Sterols}

Sterols are derived from a common structural precursor, the sterane or cyclopentanoperhydrophenanthrene, consisting in a main structure formed by four 
aromatic rings identified as A, B, C and D rings. All sterols have at carbon 3 of A ring a polar hydroxyl group being the rest of the structure non-polar, which gives them certain amphipathic character, such as phospholipids. Sterols have also a double bound at carbons 5 and 6 of ring B [58]. This double bond can be saturated (reduced) which leads to the formation of stanols, which together with plant sterols derivatives are currently used as hypocholesterolemic agents when incorporated into some functional foods. At carbon 17 (ring D) both sterols as stanols have attached an aliphatic group, consisting in a linear structure of 8,9 or 10 carbon atoms, depending on whether the sterol is from animal origin (8 carbon atoms) or from vegetable origin (9 or 10 carbon atoms) [59]. Figure 9 shows the structure of cyclopentanoperhydrophenanthrene and cholesterol. Often sterols, and less frequent stanols, have esterified the hydroxyl group of carbon 3 (ring A) with a saturated fatty acid (usually palmitic; C16:0) or unsaturated fatty acid (most frequent oleic; C18:1 and less frequent linoleic acid; C18:2. The esterification of the hydroxyl group eliminates the anphipaticity of the molecule and converts it into a structure completely non-polar. Undoubtedly among sterols cholesterol is the most important because it is the precursor of important animal metabolic molecules, such as steroid hormones, bile salts, vitamin D, and oxysterols, which are oxidized derivatives of cholesterol formed by the thermal manipulation of cholesterol and that have been identified as regulators of the metabolism and homeostasis of cholesterol and sterols in general [60].

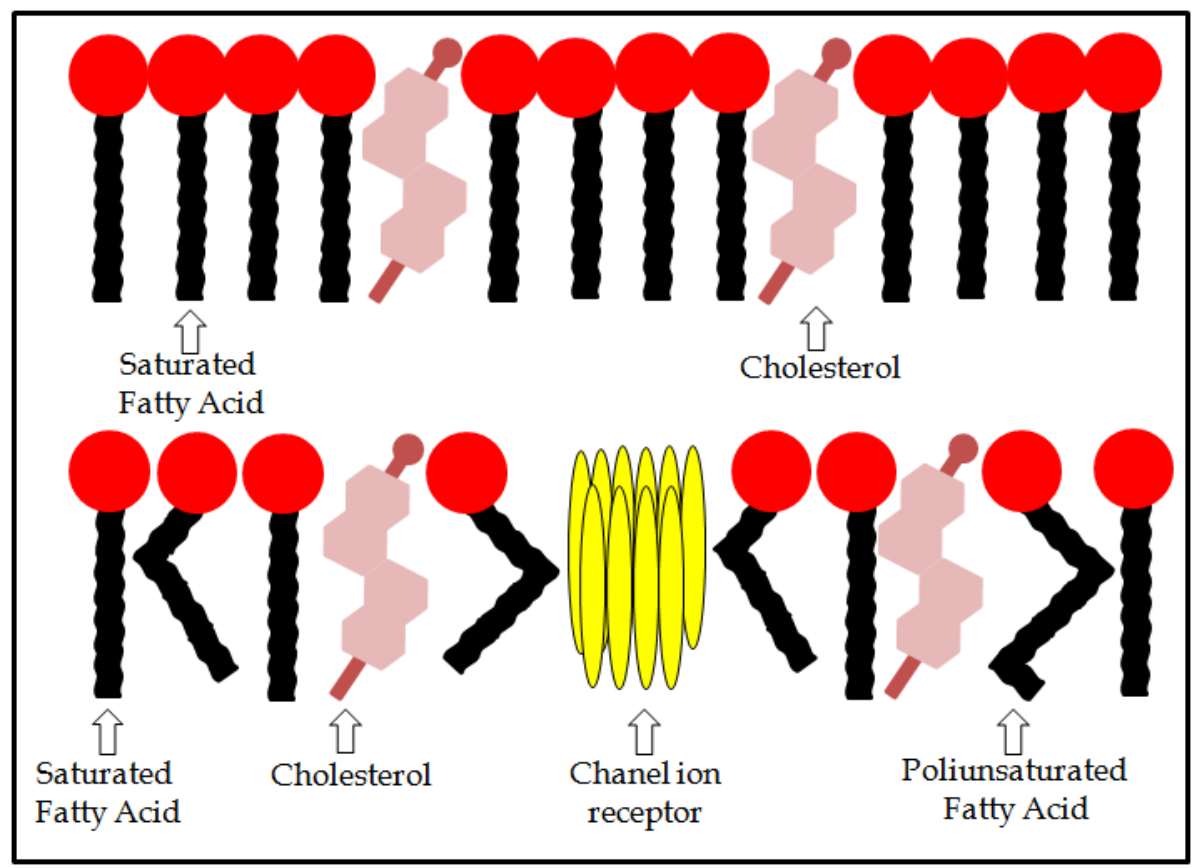

Figure 8. Simulation how the structural differences of the fatty acids which comprise phospholipids may affect the physical and chemical behavior of a membrane 


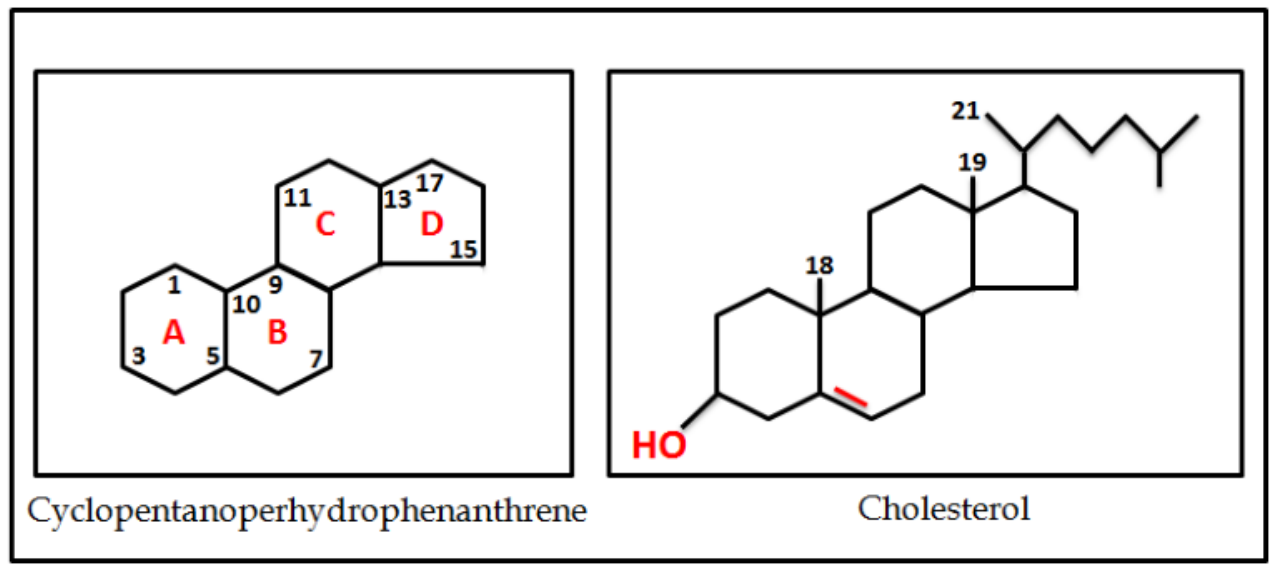

Figure 9. Structure of cyclopentanoperhydrophenanthrene and cholesterol

\section{Conclusions}

Lipids are a large and wide group of molecules that are present in all living organism and also in foods and characterized by particular physicochemical properties, such as their non polarity and their solubility in organic solvents. Some lipids, in particular fatty acids and sterols, are essential for animal and plant life. Lipids are key elements in the structure, biochemistry, physiology, and nutritional status of an individual, because are involved in: i) the cellular structure; ii) the cellular energy reserve, iii) the formation of regulatory metabolites, and; iv) in the regulation and gene expression, which directly affects the functioning of the body. Another important aspect related to lipids is their important involvement, either in the treatment and/or the origin of many diseases which can affect humans. Structural and functional characteristics of lipids, discussed in this chapter, will allow you to integrate those metabolic aspects of these important and essential molecules in close relationship of how foods containing these molecules can have a relevant influence in the health or illness of an individual.

\section{Author details}

Rodrigo Valenzuela B.

Nutrition and Dietetics School, Faculty of Medicine, University of Chile, Santiago Chile

Alfonso Valenzuela B.

Lipid Center, Nutrition and Food Technology Institute, University of Chile, Faculty of Medicine, University of Los Andes, Santiago Chile

\section{Acknowledgement}

The authors are grateful from FONDECYT, FONDEF and INNOVA-Chile the support of their research. 


\section{References}

[1] Carpenter K. (1998). Early ideas on the nutritional significance of lipids, J. Nutr. 128, 423S-426S.

[2] Orešič M. (2009). Metabolomics, a novel tool for studies of nutrition, metabolism and lipid dysfunction. Nutrition, Metabolism and Cardiovascular Diseases, Vol 19, pp. 816-24.

[3] Burlingame B, Nishida C, Uauy R. et al. (2009). Fats and fatty acids in human nutrition; joint FAO/WHO Expert Consultation. Ann. Nutr. Metab., Vol 55, pp. 1-3.

[4] Food and Agriculture Organization of the United Nations (FAO). (2010). Fats and fatty acids in human nutrition Report of an expert consultation. Food and Nutrition. Paper 91, pp. 9-19.

[5] Valenzuela A. (2009). Docosahexaenoic acid (DHA), an essential fatty acid for the proper functioning of neuronal cells: Their role in mood disorders. Grasas $\mathcal{E}$ Aceites, Vol 60, pp. 203-12.

[6] Simopoulos A. (2011), Evolutionary aspects of diet: the omega-6/omega-3 ratio and the brain. Mol Neurobiol, Vol 44, pp. 203-15.

[7] Valenzuela R, Bascuñán K, Valenzuela A, et al. (2009). Omega-3 fatty acids, neurodegenerative and psychiatric diseases: A new preventive and therapeutic approach. Rev Chil Nutr, Vol 36, pp. 1120-28.

[8] Campoy C, Escolano-Margarit M, Anjos T, et al. (2012). Omega 3 fatty acids on child growth, visual acuity and neurodevelopment. Br J Nutr. 2012, Vol 107, Suppl 2:S85-106.

[9] Weylandt K, Chiu C, Gomolka B, et al. (2012). Omega-3 fatty acids and their lipid mediators: towards an understanding of resolvin and protectinformation. Prostaglandins Other Lipid Mediat. Vol 97, pp. 73-82.

[10] Zúñiga J, Cancino M, Medina F, et al. (2011). N-3 PUFA supplementation triggers PPAR $-\alpha$ activation and PPAR $\alpha / N F-\kappa B$ interaction: anti-inflammatory implications in liver ischemia-reperfusion injury. PLoS One. Vol 6, pp. e28502.

[11] Dawson P, Lan T, and Rao A. (2009). Bile acid transporters J. Lipid Res. Vol 50, pp. 234057.

[12] Dietary fats and coronary heart disease. (2012). Willett WC. J Intern Med. Vol 272, pp. 13-24.

[13] Valenzuela R, and Videla L. (2011). The importance of the long-chain polyunsaturated fatty acid_n-6/n-3 ratio_in development of non-alcoholic fatty liver associated with obesity. Food Funct. Vol 2, pp. 644-8.

[14] Katan M, Zock P, and Mensink R. (1994). Effects of fats and fatty acids on blood lipids in humans: an overview. Am. J. Clin. Nutr., Vol 60, pp. 1017S-22S.

[15] Valenzuela A, Delplanque B, and Tavella M. (2011). Stearic acid: a possible substitute for trans fatty acids from industrial origin. Grasas $\mathcal{E}$ Aceites. Vol 62, pp. 131-38.

[16] Lee Y, Tang T, and Lai O. (2012). Health Benefits, Enzymatic Production, and Application of Medium and Long-Chain Triacylglycerol(MLCT) in Food Industries: A Review. J Food Sci. doi: 10.1111/j.1750-3841.2012.02793.x

[17] Asensio C, Nepote V, and Grosso N. (2011) Chemical stability of extra-virgin_olive oil added with oregano essential oil. J Food Sci. Vol 76, pp. S445-50. 
[18] Saggini A, Anogeianaki A, Angelucci D, et al. (2011). Cholesterol and vitamins: revisited study. J Biol Regul Homeost Agents. Vol 25, pp. 505-15.

[19] Fahy E, Subramanium S, Brown A, et al. (2005). A comprehensive classification system for lipids. J. Lipid Res. Vol 46, pp. 839-61.

[20] Kuipers R, Luxwolda M, Offringa P, et al. (2012). Fetal intrauterine whole body linoleic, arachidonic and docosahexaenoic acid contents and accretion rates. Prostaglandins Leukot Essent Fatty Acids. Vol 86, pp 13-20.

[21] Rodríguez E, Giri M, Rottiers $R$ et al. Fatty acid composition of erythrocyte phospholipids is related to insulin levels, secretion and resistance in obese type 2 diabetics on Metformin. Clinica Chimica Acta, Vol 346. pp. 145-52.

[22] Gurr MI. (1992). Dietary lipids and coronary heart desease: Old evidence, new persspective Prog in Lip Res. Vol 31, pp 195-243.

[23] Martin D, Muriel E, Antequera T, et al. Quantitative changes in the fatty acid profile of lipid fractions of fresh loin from pigs as affected by dietary conjugated linoleic acid and monounsaturated fatty acids during refrigerated storage. J. Food Composition and Analysis. Vol 22, pp. 102-11.

[24] Arnauld S, Fidaleo M, Clémencet MC, et al. (2009). Modulation of the hepatic fatty acid pool in peroxisomal 3-ketoacyl-CoA thiolase B-null mice exposed to the selective PPARalpha agonist Wy14,643. Biochimie. Vol 91, pp. 1376-86.

[25] Harris W, Miller M, Tighe A, et al. (2008). Omega-3 fatty acids and coronary heart disease risk: Clinical and mechanistic perspectives Atherosclerosis, Vol 197, pp. 12-24.

[26] Osorio N, Ribeiro M, da Fonseca M, et al. (2008). Interesterification of fat blends rich in $\omega-3$ polyunsaturated fatty acids catalysed by immobilized Thermomyces lanuginosa lipase under high pressure J Mol Catalysis B: Enzymatic, Vol 52. pp. 58-66.

[27] Skerratt J, Nichols P, Bowman J, et al. (1992). Occurrence and significance of long-chain $(\omega-1)$-hydroxy fatty acids in methane-utilizing bacteria. Organic Geochemistry. Vol 18, pp. 189-94.

[28] Ansari G, Bhupendra S, Kaphalia M, et al. (1995). Fatty acid conjugates of xenobiotics Toxicology Letters. Vol 75, pp. 1-17.

[29] Michl J, and West R. Conformations of linear chains. (2000). Systematics and suggestions for nomenclature. Acc Chem Res. Vol 33, pp. 821-3.

[30] Din J, Newby D, and Flapan A, (2004). Omega 3 fatty acids and cardiovascular disease-fishing for a natural treatment. BMJ. Vol 3, pp. 30-35.

[31] Kris-Etherton P, Taylor D, Yu-Poth S, et al. (2000). Polyunsaturated fatty acids in the food chain in the United States. Am J Clin Nutr. Vol 71. pp. S179-88.

[32] Kris-Etherton P, Harris W, Appel LJ for the Nutrition Committee. (2002). AHA scientific statement. Fish consumption, fish oil, omega-3 fatty acids, and cardiovascular disease. Circulation. Vol 106. pp. 2747-57.

[33] Kishi T, Carvajal O, Tomoyori H, et al.(2002). Structured triglycerides containing medium-chain fatty acids and linoleic acid differently influence clearance rate in serum of triglycerides in rats Nutrition Research, Vol 22, pp. 1343-51.

[34] Carpentier Y, Simoens C, Siderova V, et al. (1997). Recent developments in lipid emulsions: relevance to intensive care. Nutrition, Vol 13, pp. 73-78. 
[35] Irene Cetin, Gioia Alvino, Manuela Cardellicchio. (2009). Long chain fatty acids and dietary fats in fetal nutrition. J Physiol. Vol 15, pp. 3441-51.

[36] Rekha S, Singhal A, Gupta P, et al. Low-calorie fat substitutes. Trends in Food Science $\mathcal{E}$ Technology. Vol 2, pp. 241-44.

[37] Morita O, Knapp J, Tamaki Y, et al. (2008). Safety assessment of dietary diacylglycerol oil: A two-generation reproductive toxicity study in rats. Food and Chemical Toxicology, Vol 46, pp. 3059-68.

[38] Blasbalg T, Hibbeln J, Ramsden C, et al. (2011). Changes in consumption of omega-3 and omega-6 fatty acids in the United States during the 20th century. Am J Clin Nutr. Vol 93, pp. 950-62.

[39] Le H, Meisel J, de Meijer V, et al. (2012). The essentiality of arachidonic acid and docosahexaenoic acid. Prostaglandins Leukot Essent Fatty Acids. Vol 81, pp. 165-170.

[40] Valenzuela A, and Morgado N. (1999). Trans fatty acid isomers in human health and in the food industry. Biol Res. Vol 32, pp. 273-87.

[41] Micha R, King I, Lemaitre R, et al. (2010). Food sources of individual plasma phospholipid trans fatty acid isomers: the Cardiovascular Health Study. Am J Clin Nutr. Vol 91, pp. 883-93.

[42] Minville-Walz M, Gresti J, Pichon L, et al. (2012). Distinct regulation of stearoyl-CoA desaturase 1 gene expression by cis and trans C18:1 fatty acids in human aortic smooth muscle cells. Genes Nutr. Vol 7, pp. 209-16.

[43] Valenzuela A. (2008). Trans fatty acids I. Origin and effects in human health. Rev Chil Nutr. Vol 35, pp. 162-71.

[44] Micha R, and Mozaffarian D. (2008). Trans Fatty Acids: Effects on Cardiometabolic Health and Implications for Policy. Prostaglandins Leukot Essent Fatty Acids. Vol 79, pp. 147-52.

[45] Kuhnt K, Baehr M, Rohrer C, et al. (2011). Trans fatty acid isomers and the trans9/trans-11 index in fat containing foods. Eur J Lipid Sci Technol. Vol 113, pp. 1281-92.

[46] Seppänen-Laakso T, Laakso I, Backlund P, et al. (1996). Elaidic and trans-vaccenic acids in plasma phospholipids as indicators of dietary intake of 18:1 trans-fatty acids. Journal of Chromatography B: Biomedical Sciences and Applications. Vol 687, pp. 996, Pages 371-78.

[47] Dilzer A, and Park Y. (2012). Implication of conjugated linoleic acid (CLA) in human health. Crit Rev Food Sci Nutr. Vol 52, pp. 488-513.

[48] Izadifar M. (2005). Neural network modeling of trans isomer formation and unsaturated fatty acid changes during vegetable oil hydrogenation. Journal of Food Engineering. Vol 66, pp. 227-32.

[49] Green J, Liu Z, and Bazinet P. (2010). Brain phospholipid arachidonic acid half-lives are not altered following 15 weeks of N-3 polyunsaturated fatty acid_adequate or deprived diet. J Lipid Res. Vol 51, pp. 535-43.

[50] Koga Y, and Goldfine H. (1984). Biosynthesis of_phospholipids_in Clostridium butyricum: kinetics of synthesis of plasmalogens and the glycerol acetal of ethanolamine plasmalogen. J Bacteriol. Vol 159, pp 597-604.

[51] García-Sáinz J, and Fain J. (1980). Effect of insulin, catecholamines and calcium ions on phospholipidmetabolism in isolated white fat-cells. Biochem J. Vol 15, pp. 781-89. 
[52] Castro-Perez J, Roddy T, Nibbering N, et al. (2011). Localization of Fatty Acyl and Double Bond Positions in_Phosphatidylcholines_Using a Dual Stage CID Fragmentation Coupled with Ion Mobility Mass Spectrometry. J Am Soc Mass Spectrom. Vol 22, pp. 1552-67.

[53] Huang Z, Jiang J, Tyurin V, et al. (2009). Cardiolipin_deficiency leads to decreased cardiolipin peroxidation and increased resistance of cells to apoptosis. Free Radic Biol Med. Vol 44, pp. 1935-44.

[54] Oleic- and Docosahexaenoic Acid-Containing_Phosphatidylethanolamines_Differentially Phase Separate from Sphingomyelin. Shaikh S, LoCascio D, Soni S, et al. (2010). Biochim Biophys Acta. Vol 1788, pp 2421-26.

[55] Esquenazi S, and Bazan H. (2010). Role of_Platelet-Activating Factor_in Cell Death Signaling in the Cornea: A Review. Mol Neurobiol. Vol 42, pp. 32-38.

[56] Gimenez M, Oliveros L, and Gomez N. (2011). Nutritional Deficiencies and Phospholipid Metabolism. Int J Mol Sci. Vol 12, pp 2408-33.

[57] Levantesi G, Silletta M, and Marchioli R. (2010). Uses and benefits of omega-3 ethyl esters in patients with cardiovascular disease. J Multidiscip Healthc. Vol 3, pp. 79-96.

[58] Gulati S, Liu Y, Munkacsi A, et al. (2011). Sterols and sphingolipids: Dynamic duo or partners in crime?. Prog Lipid Res. Vol 49, pp. 353-65.

[59] Izar M, Teganiv D, Kasmas S, et al. (2010). Phytosterols and phytosterolemia: gene-diet interactions Genes Nutr. Vol 6, pp. 17-26.

[60] Björkhem I. (2009). Are side-chain oxidized oxysterols regulators also in vivo?. J Lipid Res. Vol 50, pp. S213-18. 